\title{
Tunneling microscope observation of a structural surface phase transition: Structure, fluctuations, and local effects
}

\section{Citation}

Hwang, Ing-Shouh, and Jene A. Golovchenko. 1993. Tunneling Microscope Observation of a Structural Surface Phase Transition: Structure, Fluctuations, and Local Effects. Physical Review Letters 71, no. 2: 255-258. doi:10.1103/physrevlett.71.255.

\section{Published Version}

doi:10.1103/PhysRevLett.71.255

\section{Permanent link}

http://nrs.harvard.edu/urn-3:HUL.InstRepos:29407029

\section{Terms of Use}

This article was downloaded from Harvard University's DASH repository, and is made available under the terms and conditions applicable to Other Posted Material, as set forth at http:// nrs.harvard.edu/urn-3:HUL.InstRepos:dash.current.terms-of-use\#LAA

\section{Share Your Story}

The Harvard community has made this article openly available.

Please share how this access benefits you. Submit a story.

\section{Accessibility}




\title{
Tunneling Microscope Observation of a Structural Surface Phase Transition: Structure, Fluctuations, and Local Effects
}

\author{
Ing-Shouh Hwang ${ }^{1}$ and Jene A. Golovchenko ${ }^{1,2,3}$ \\ ${ }^{1}$ Division of Applied Sciences, Harvard University, Cambridge, Massachusetts 02138 \\ ${ }^{2}$ Department of Physics, Harvard University, Cambridge, Massachusetts 02138 \\ ${ }^{3}$ Rowland Institute for Science, Cambridge, Massachusetts 02142 \\ (Received 19 January 1993)
}

\begin{abstract}
We study a reversible phase transition of one monolayer of $\mathrm{Pb}$ on a $\mathrm{Ge}(111)$ surface with a tunneling microscope over a temperature range from below to above the transition $\left(\sim 175^{\circ} \mathrm{C}\right)$. The controversial high temperature phase is actually a simple $\mathrm{Pb}$-terminated $1 \times 1$ structure. Below the transition temperature, $\mathrm{Pb}$ atoms are displaced from their $T_{1}$ positions and form trimers with $\sqrt{3} \times \sqrt{3}$ periodicity. A fluctuating structure with patches of trimers can be resolved slightly above the transition temperature. The important effects of boundaries and domain size on the phase transformation are discussed.
\end{abstract}

PACS numbers: $61.16 . \mathrm{Ch}, 68.35 . \mathrm{Rh}, 68.55 . \mathrm{Jk}, 68.55 . \mathrm{Nq}$

The study and characterization of phase transitions is a central theme of modern condensed matter physics. Historically, macroscopic thermodynamic properties and diffraction measurements, as a function of the appropriate intrinsic thermodynamic variables, have provided the experimental foundation upon which the development of theoretical models and methods have depended. Symmetry and dimensionality play a crucial role, with twodimensional (2D) phase transformations expected to be more strongly influenced by fluctuations than those in three dimensions. The search for $2 \mathrm{D}$ systems has naturally led to an experimental focus on structural transformations that occur on crystal surfaces. Because of the small number of atoms involved $\left(\sim 10^{15} \mathrm{~cm}^{-2}\right)$ and the interference from the bulk crystal, traditional methods of studying phase transitions at surfaces can face major obstacles in presenting a complete picture of the essential atomic scale phenomena whose macroscopic consequences theory endeavors to explain. On the other hand, the rich array of interesting and technologically important phenomena on surfaces has recently provided additional motivation for intensive study of surfaces and stimulated the development of new and powerful tools like the scanning tunneling microscope (STM).

In the following we demonstrate how real space images from a tunneling microscope can provide the crucial information required for the characterization and understanding of a phase transition which has eluded previous studies. There has been recent progress in this direction owing to the development of tunneling microscopes that operate at high temperatures. Surface phase transitions of the semiconductor $\mathrm{Si}(111)-(7 \times 7)$ [1] and $\mathrm{Ge}(111)$ $c(2 \times 8)$ [2] have been studied. In both cases, however, atomic motions were too fast for the structure on the high temperature side of the transition to be resolved. More recently, a coverage-dependent (rather than temperature-dependent) study on a metal surface has provided a detailed view of the disordering transition of $\mathrm{Cs}$ on $\mathrm{Cu}(110)$ [3]. Here we present a temperature-driven phase transition study of $\mathrm{Pb}$ on $\mathrm{Ge}(111)$ at monolayer coverage. The literature shows that, in spite of rather intense study, this low temperature $\left(-175^{\circ} \mathrm{C}\right)$ phase transition was not understood. We will show, by resolving high and low temperature phases, that it is an order-order transition, which actually allows atomic spatial and temporal fluctuations to be observed just above the transition temperature. The observed symmetry suggests the system is related to the three-state Potts model. In addition, the influence of strain, phase boundaries, and finite size domains can be observed.

$\mathrm{Pb} / \mathrm{Ge}$ (111) has been studied for years [4-11]. Bulk $\mathrm{Pb}$ and $\mathrm{Ge}$ do not intermix and $\mathrm{Pb}$ atoms do not evaporate from the surface for temperatures below $350^{\circ} \mathrm{C}$. Thus, $\mathrm{Pb}$ atoms remain at the surface after the deposition. Very importantly, $\mathrm{Pb}$ and $\mathrm{Ge}$ atoms are easily distinguished in tunneling microscope images [10]. There are two different $\sqrt{3} \times \sqrt{3}$ structures which occur before the completion of the first $\mathrm{Pb}$ overlayer on the $\mathrm{Ge}(111)$ surface [4-9]. The $\frac{1}{3}$ monolayer (ML) low coverage $\alpha$ phase has been found to resemble many other systems of metals on semiconductor (111) surfaces. That is, one adatom adsorbs on a $T_{4}$ site for each $\sqrt{3} \times \sqrt{3}$ unit cell. The high coverage phase shows a reversible phase transition from a different $\sqrt{3} \times \sqrt{3}$ phase (the $\beta$ phase) to a $1 \times 1$ phase at about $175^{\circ} \mathrm{C}$, as observed by low energy electron diffraction (LEED), reflection high energy electron diffraction (RHEED), and x-ray scattering [4-8]. However, controversy and confusion about the exact coverage and atomic structures for the $\beta$ and the high temperature $1 \times 1$ phases still persist. Diffuse rods in RHEED and a ring of diffuse scattering in $x$-ray diffraction studies in the high temperature $1 \times 1$ phase led Ichikawa [4] and Grey et al. [5] to conclude that it is a 2D liquid. However, LEED studies do not show any rings of diffuse scattering [6]. Also, the results of an $x^{-}$ ray standing-wave study are not consistent with the model of a 2D liquid; rather, the high temperature phase was suggested to be composed of small islands of the original $\beta-\sqrt{3} \times \sqrt{3}$ crystalline overlayer [7].

In order to resolve these issues, we have done extensive 
studies of this surface using a tunneling microscope. Our experiments were performed in an ultrahigh vacuum chamber with a base pressure of $6 \times 10^{-11}$ torr. $\mathrm{Pb}$ was deposited from an effusion cell onto a clean $\mathrm{Ge}(111)$ $c(2 \times 8)$ surface, whose preparation and structure have been described elsewhere [10,11]. After identifying the surface structure and the phase transition with LEED, the sample was transferred to the STM stage for imaging. At $\frac{1}{3} \mathrm{ML}\left(1 \mathrm{ML}=7.22 \times 10^{14} \mathrm{~cm}^{-2}\right)$, the low coverage $\sqrt{3} \times \sqrt{3}$ phase (the $\alpha$ phase, with $\mathrm{Pb}$ atoms on $T_{4}$ sites) is observed in our STM images. Above $\frac{1}{3} \mathrm{ML}$, domains of the high coverage $\beta-\sqrt{3} \times \sqrt{3}$ phase are found. Most of them nucleate at step edges on the lower terraces. To observe the phase transition of the $\beta$ phase, we image the surfaces at sample temperatures from $24^{\circ} \mathrm{C}$ up to $200^{\circ} \mathrm{C}$ (with thermocouple accuracy of $\pm 5^{\circ} \mathrm{C}$ and precision of $\sim 0.2^{\circ} \mathrm{C}$ ).

Figure 1 shows a tunneling image of the high coverage phase taken at $168^{\circ} \mathrm{C}$ with a tip bias of $+0.8 \mathrm{~V}$ (the transition temperature is $\sim 172^{\circ} \mathrm{C}$ ). The upper part of the figure is the $\beta$ phase, which may be viewed as resulting from a periodic array of $\mathrm{Pb}$ trimers, one per $\sqrt{3} \times \sqrt{3}$ unit cell. The trimers are centered on $\mathrm{H}_{3}$ sites, which can be determined by extrapolating from the well-understood $\alpha$ phase on an image that contains both $\alpha$ and $\beta$ phases (not shown) [12]. Figure 2 shows a simple atomic model of the $\beta$ phase, which is different from previous models [4-9]. Each $\mathrm{Pb}$ atom is displaced from the $T_{1}$ site above the first layer Ge atom, to which it is bonded, towards the threefold hollow $\mathrm{H}_{3}$ site. The resulting trimers are centered on $\mathrm{H}_{3}$ sites. Trimers centered on $T_{4}$ sites would be rotated $60^{\circ}$ and none is observed in our images. The spacing between $\mathrm{Pb}$ atoms in the trimer is $3.5( \pm 0.3) \AA$

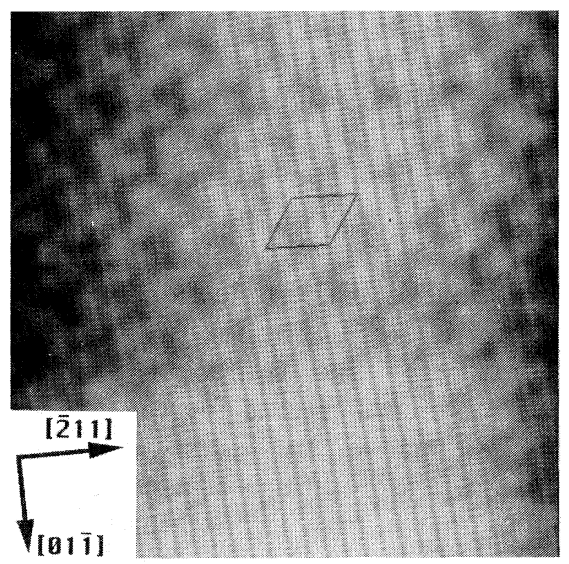

FIG. 1. $55 \AA \times 70 \AA$ tunneling image of the high coverage phase taken at $168^{\circ} \mathrm{C}$. The $\beta$ phase is seen in the upper part of the image and a $1 \times 1$ phase, which is the same as the high temperature phase, is present in the lower part. The $\alpha$ phase lies just below the boundary of the image. The corrugation in the image is $\sim 0.2 \AA$. A unit cell of the $\beta$ phase and crystal directions are indicated. (note that the interatomic spacing in bulk $\mathrm{Pb}$ is $3.5 \AA$ ). Clearly, the formation of $\mathrm{Pb}$ trimers in the $\beta$ phase is due to the attractive interaction between neighboring $\mathrm{Pb}$ atoms, and the displacement of each $\mathrm{Pb}$ atom from its ideal $T_{1}$ sites is $0.3( \pm 0.2) \AA$ [13]. Since the atomic model of the $\beta$ phase is established, we anticipate that a more accurate value for this atomic displacement can be obtained using $x$-ray scattering techniques.

There has been a debate about whether the saturation coverage of the $\beta$ phase is 1 or $\frac{4}{3}$ ML [4-9]. The coverage can be seen to be 1 ML in our tunneling images since there are three $\mathrm{Pb}$ atoms in each $\sqrt{3} \times \sqrt{3}$ unit cell. Additionally, a surface imaged over an area of $1.3 \times 10^{7} \AA^{2}$ with the STM was found to be covered with $81( \pm 4) \% \beta$ phase and $19( \pm 4) \% \alpha$ phase. This corresponds to $0.87( \pm 0.03$ ) $\mathrm{ML}$ of $\mathrm{Pb}$ assuming the coverage of the $\beta$ phase is $1 \mathrm{ML}$, and $1.14( \pm 0.04) \mathrm{ML}$ assuming $\frac{4}{3} \mathrm{ML}$. The $\mathrm{Pb}$ coverage of the sample was measured to be 0.91 ( \pm 0.09 ) ML using Rutherford backscattering. This provides additional evidence that the saturation coverage of the $\beta$ phase is $1 \mathrm{ML}$.

At room temperature, only the $\beta$ phase is seen across the entire domain of the high $\mathrm{Pb}$ coverage phase. However, near the bottom of the $168^{\circ} \mathrm{C}$ image shown in Fig. 1, a $1 \times 1$ phase, with bright spots centered on the $T_{1}$ sites, appears in the vicinity of the $\alpha$ phase, which lies below the boundary of the image. This $1 \times 1$ phase is identical to the one observed well above the transition temperature, when no $\beta$ phase remains. Therefore, the transformation starts from the boundary with the $\alpha$ phase.

Above the transition temperature, the long range $\sqrt{3}$ $\times \sqrt{3}$ order is destroyed by thermal fluctuations. Figure 3 shows a remarkable image obtained at $173^{\circ} \mathrm{C}$ (just above the transition temperature) with a tip bias of $+0.4 \mathrm{~V}$. The surface is in a state of wild, though resolvable, fluc-

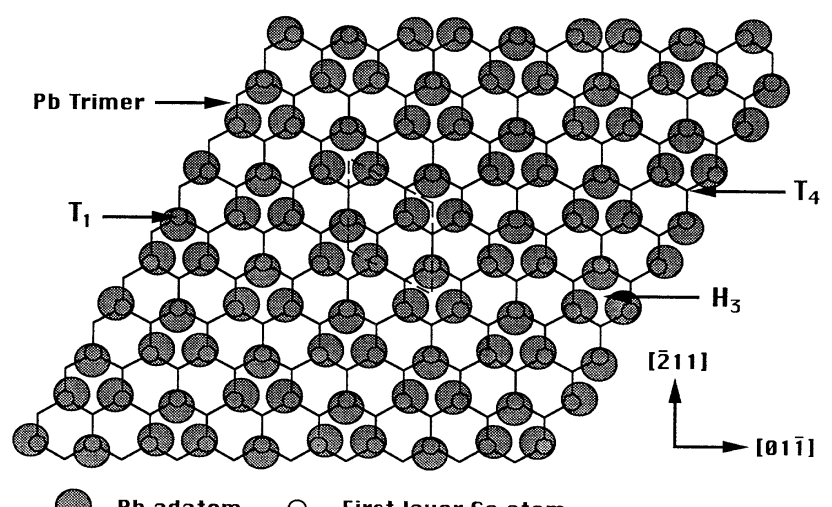

Pb adatom $\bigcirc$ First layer Ge atom

FIG. 2. Simple atomic model of the $\beta$ phase on the Ge(111) surface. Each $\mathrm{Pb}$ atom is bonded to the $\mathrm{Ge}$ atom underneath, and is displaced by about $0.3 \AA$ (not to the scale in the figure) from the $T_{1}$ site toward an $H_{3}$ site to form a trimer with two other neighboring $\mathrm{Pb}$ atoms. A unit cell and crystal directions are indicated. 


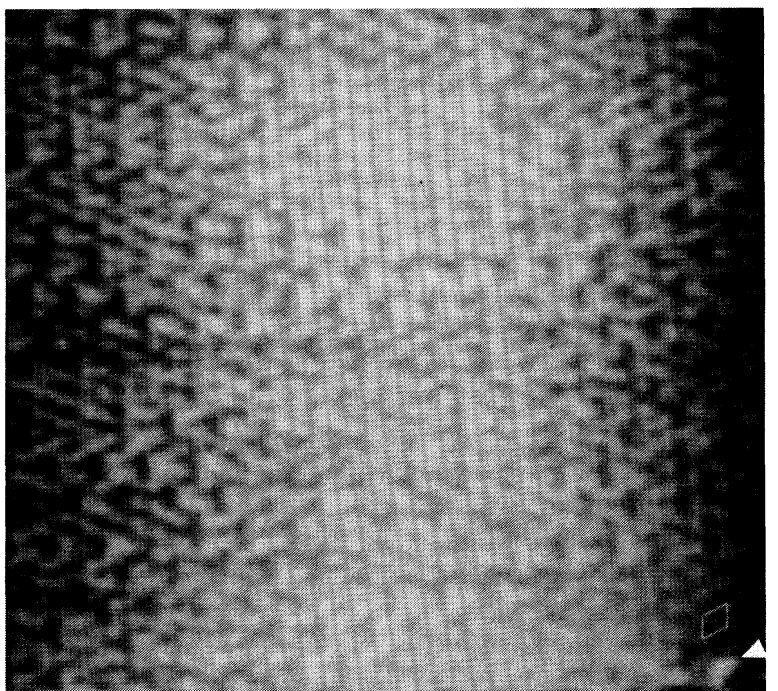

FIG. 3. $110 \AA \times 110 \AA$ tunneling image of the high coverage phase taken at $173^{\circ} \mathrm{C}$, just above the transition temperature. Short-range order with patches of trimers centered on different sets of $\mathrm{H}_{3}$ sites can be seen. At the lower right corner is a boundary with the $\alpha$ phase (indicated by an arrow), and a $1 \times 1$ structure (with a unit cell indicated) can be seen close to it. The crystal directions are the same as those in Fig. 1.

tuation. A structure with patches of trimers can be clearly seen. A $1 \times 1$ structure is also visible in the vicinity of a phase boundary (indicated by an arrow). This image is composed of 180 horizontal scan lines, each of which takes $0.43 \mathrm{~s}$.

A study of Fig. 2 shows that there can be three possible domains of $\beta-\sqrt{3} \times \sqrt{3}$, translationally inequivalent, supported by the underlying lattice. To obtain the two additional domains, simply shift all the trimers so that they are centered on either set of unoccupied $\mathrm{H}_{3}$ sites. Figure 4 shows an atomic model of a snapshot of the high temperature phase, which contains small fluctuating patches of each domain separated by dimers and monomers at the boundaries. Since a $\mathrm{Pb}$ atom can be displaced in any one of the three possible directions to form a trimer with neighboring $\mathrm{Pb}$ atoms, this system should be closely related to the three-state Potts model [14] although the system we study is clearly not homogeneous. As the temperature increases, the average size of a patch decreases and fluctuations become faster. Each $\mathrm{Pb}$ atom, on the average, is then centered over the Ge atom underneath. This explains the observation of the ordered $1 \times 1$ structure in the STM images several degrees above the transition temperature. This high temperature phase clearly is not the 2D liquid reported by earlier $x$-ray scattering and RHEED studies.

Since both temporal and spatial fluctuations are contained in Fig. 3, quantitative analysis of these types of images will yield detailed temperature-dependent correlation lengths and times that characterize the phase transi-

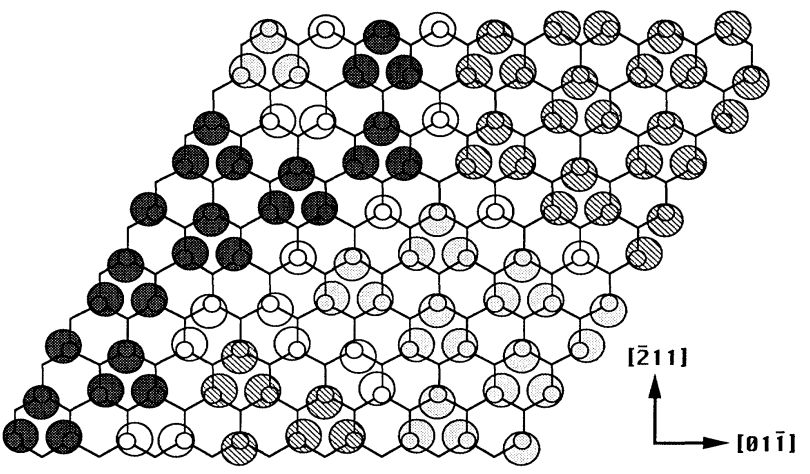

Pb adatoms $\bigcirc$ First layer Ge atom

FIG. 4. Model of one possible atomic configuration of the fluctuating high temperature phase. Shading is used to indicate domains of trimers centered on different sets of $\mathrm{H}_{3}$ sites.

tion. However, this requires good statistics, more precise temperature measurement, and faster scanning, which will be the next goal.

The transition of the $\beta$ phase to the $1 \times 1$ phase starting from the boundary with the $\alpha$ phase may be due to the fact that the inhomogeneous surface strain field originating from the $\alpha$ phase lowers the transition temperature. We have also observed that small regions of $\beta-\sqrt{3} \times \sqrt{3}$ bounded by the $\alpha$ phase transform into $1 \times 1$ at lower temperatures than larger regions. However, quantitative measurement and interpretation are difficult because defects and step edges at the boundary of the high coverage phase can also affect the transition temperature (perhaps due to surface strain fields, conditions on the boundaries, etc.) Between $\frac{1}{3} \mathrm{ML}$ and close to $1 \mathrm{ML}$ ( $\left.\geq 0.9 \mathrm{ML}\right)$, the transition temperature increases only a few degrees with increasing $\mathrm{Pb}$ coverage. Close to $1 \mathrm{ML}$, new local effects cause a sharp increase in the transition temperature (to $\sim 300^{\circ} \mathrm{C}$ ). The results of $\mathrm{Pb}$ coverage near and higher than $1 \mathrm{ML}$ will be presented in our future full report of the phase transition.

To conclude, we have presented what we believe to be the most comprehensive tunneling microscope study of a structural surface phase transition to date. The phases below and above the transition temperature are resolved on the atomic scale, the strongly fluctuating region just above the transition is exposed, and surface strain fields originating from another reconstruction are also found to affect the phase transition. The inhomogeneity of the strain fields and finite size domains observed on the surface suggest that great care should be taken in the interpretation of structural phase transitions using nonmicroscopic techniques.

We would like to thank Dr. Robert Martinez, Silva K. Theiss, Professor Efthimios Kaxiras, Professor Frans Spaepen, Tom Chou, and Professor David Nelson for fruitful discussions and criticism of this manuscript. This 
research was supported by the Materials Research Laboratory at Harvard University (Contract No. NSFDMR-8920490), the Office of Naval Research (Contract No. N00014-90-J-1234), and the Joint Services Electronics Program (Contract No. N00014-89-J-1023).

[1] S. Kitamura, T. Sato, and M. Iwatsuki, Nature (London) 351, 215 (1991).

[2] R. M. Feenstra, A. J. Slavin, G. A. Held, and M. A. Lutz, Phys. Rev. Lett. 66, 3257 (1991).

[3] R. Schuster, J. V. Barth, G. Ertl, and R. J. Behm, Phys. Rev. Lett. 69, 2547 (1992).

[4] T. Ichikawa, Solid State Commun. 49, 59 (1984); 46, 827 (1983).

[5] F. Grey, R. Feidenhans'l, J. Skov Pedersen, M. Nielsen, and R. L. Johnson, Phys. Rev. B 41, 9519 (1990).

[6] J. J. Metois and G. Le Lay, Surf. Sci. 133, 422 (1983).

[7] B. N. Dev, F. Grey, R. L. Johnson, and G. Materlik, Europhys. Lett. 6, 311 (1988).

[8] F. Grey, Ph.D. thesis, Ris $\emptyset-M-2737,1988$ (unpublished).

[9] R. Feidenhans'l, J. S. Pedersen, M. Nielsen, F. Grey, and R. L. Johnson, Surf. Sci. 178, 927 (1986).

[10] E. Ganz, S. K. Theiss, I.-S. Hwang, and J. Golovchenko, Phys. Rev. Lett. 68, 1567 (1992); I.-S. Hwang and J.
Golovchenko, Science 258, 1119 (1992).

[11] R. S. Becker, J. A. Golovchenko, and B. S. Swartzentruber, Phys. Rev. Lett. 54, 2678 (1985); R. S. Becker, B. S. Swartzentruber, J. S. Vickers, and T. Klitsner, Phys. Rev. B 39, 1633 (1989).

[12] At low biases, $\angle 1 \mathrm{~V}$ in both polarities, where the atomic structure of the $\beta$ phase can be resolved, the tip is usually not stable above the $\alpha$ phase. Therefore, the images shown here do not contain the $\alpha$ phase.

[13] The trimers shown in Fig. 1 do not appear as perfect as the model shown in Fig. 2. We cannot tell whether the trimers have any slight deviation from the threefold symmetry which we infer from the diffraction pattern. Because very high resolution is required to resolve the fine structure of the trimers, any imperfection in the tunneling tip can affect the image. Various biases have been tried and the trimer structure similar to that shown in Figs. 1 and 3 have been consistently observed. The slight deviation mentioned above varies from tip to tip.

[14] E. Bauer, in Structure and Dynamics of Surfaces II, edited by W. Schommers and P. von Blanckenhagen (Springer-Verlag, New York, 1987), p. 115; R. J. Baxter, Exactly Solved Models in Statistical Mechanics (Academic, New York, 1982); F. Y. Wu, Rev. Mod. Phys. 54, 235 (1982); Y. Saito, J. Phys. A 15, 1885 (1982); I. G. Enting and F. Y. Wu, J. Stat. Phys. 28, 351 (1982). 


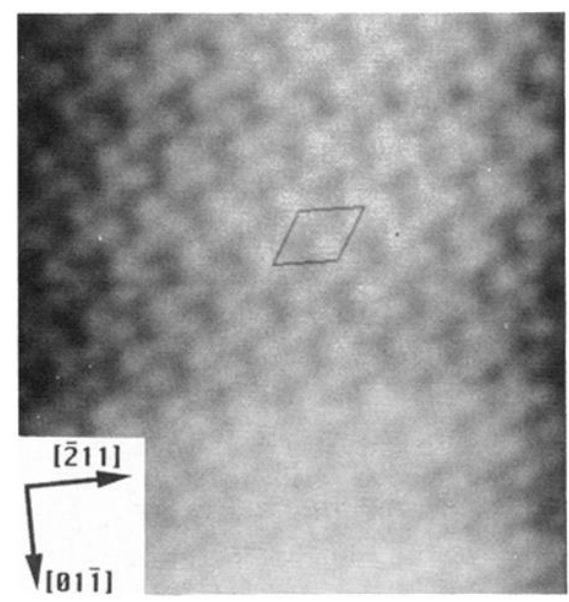

FIG. 1. $55 \AA \times 70 \AA$ tunneling image of the high coverage phase taken at $168^{\circ} \mathrm{C}$. The $\beta$ phase is seen in the upper part of the image and a $1 \times 1$ phase, which is the same as the high temperature phase, is present in the lower part. The $\alpha$ phase lies just below the boundary of the image. The corrugation in the image is $\sim 0.2 \AA$. A unit cell of the $\beta$ phase and crystal directions are indicated. 


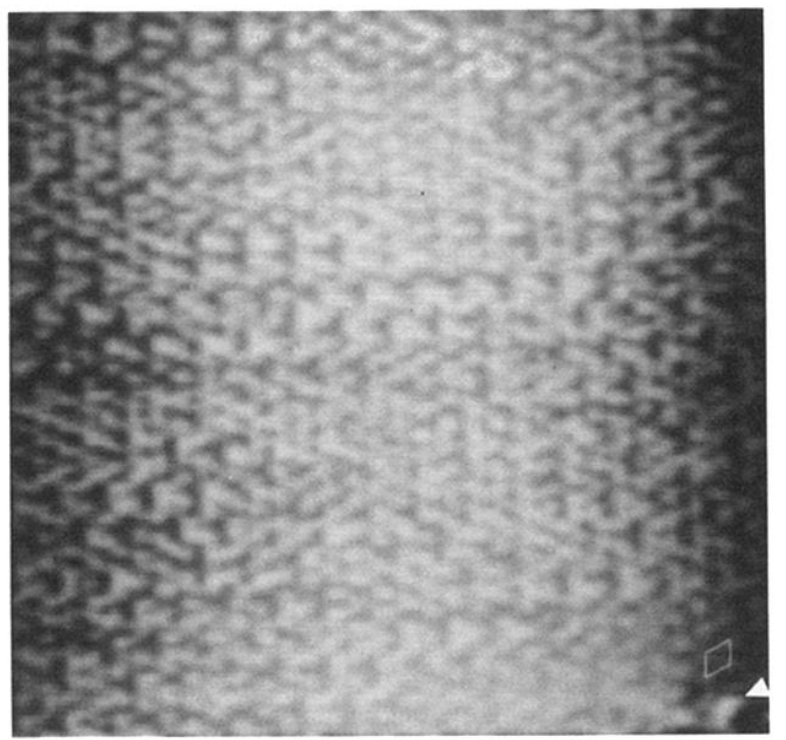

FIG. 3. $110 \AA \times 110 \AA$ tunneling image of the high coverage phase taken at $173^{\circ} \mathrm{C}$, just above the transition temperature. Short-range order with patches of trimers centered on different sets of $\mathrm{H}_{3}$ sites can be seen. At the lower right corner is a boundary with the $\alpha$ phase (indicated by an arrow), and a $1 \times 1$ structure (with a unit cell indicated) can be seen close to it. The crystal directions are the same as those in Fig. 1. 